\title{
Traditional medicine usage among adult women in Ibadan, Nigeria: a cross-sectional study
}

\author{
Suellen $\mathrm{Li}^{1 *}$, Stella Odedina ${ }^{2}$, Imaria Agwai ${ }^{2}$, Oladosu Ojengbede ${ }^{2}$, Dezheng Huo ${ }^{3}$ and Olufunmilayo I. Olopade ${ }^{4}$
}

\begin{abstract}
Background: Previous research has revealed high rates of traditional medicine usage in Nigeria. Reports of widespread contamination of herbal medicine products and higher rates of noncompliance with Western medications among traditional medicine users have raised concerns about the safety of traditional medicine use. Few studies have explored how demographic factors predict rates of traditional medicine use in the general population.

Methods: We conducted interviews of 748 adult women recruited from the communities in the city of Ibadan, Nigeria from 2013 to 2015. A structured questionnaire was created to collect data on rates of traditional medicine use and demographic factors such as age, education, ethnicity, and occupation. Multivariate logistic regressions were run to examine factors related to traditional medicine use, and the effects were measured with odds ratios (OR) along with $95 \%$ confidence interval $(95 \% \mathrm{Cl})$.

Results: The overall proportion of traditional medicine use was $81.6 \%$. Women from the Ibo and Hausa ethnic groups were significantly less likely to use traditional medicine than the majority Yoruba group $(\mathrm{OR} 0.25,95 \% \mathrm{Cl}$ $0.10-0.63$; OR $0.43,95 \% \mathrm{Cl} 0.24-0.76$ ) respectively). In addition, educated women were less likely than their noneducated counterparts to have used traditional medicine, with the biggest effect seen in women with a secondary education (OR 0.42, 95\%Cl 0.21-0.85).

Conclusions: We found a high rate of traditional medicine usage, consistent with that found in prior research. A novel finding was the significance of ethnicity as a predictor for usage rates.
\end{abstract}

Keywords: Traditional medicine, Herbal medicine, Global Health, Women's health, Ethnic minorities

\section{Background}

The World Health Organization (WHO) estimates that $80 \%$ of the world population relies on complementary and alternative medicines (CAM), or traditional medicine, which includes all healing practices indigenous to different cultures. Traditional medicine is often juxtaposed with modern biomedicine. In Nigeria and other

\footnotetext{
* Correspondence: sli1@mgh.harvard.edu

'Department of Internal Medicine, Massachusetts General Hospital, Boston, MA, USA

Full list of author information is available at the end of the article
}

parts of West Africa, herbal remedies and spiritual healing are particularly common [1]. Reported uses for traditional medicine (TM) include cancer [2], diabetes [3], HIV [4], and hypertension [5], among others. Parents often treat their children with traditional medicine, with documented uses for epilepsy, asthma, and sickle cell disease [6]. These practices are not confined to their country of origin, however, and immigrants from Nigeria often bring their traditional beliefs to their new country of residence, where physicians are unfamiliar with their practices [7]. This is especially important in oncology, as

C C The Author(s). 2020 Open Access This article is licensed under a Creative Commons Attribution 4.0 International License, which permits use, sharing, adaptation, distribution and reproduction in any medium or format, as long as you give appropriate credit to the original author(s) and the source, provide a link to the Creative Commons licence, and indicate if changes were made. The images or other third party material in this article are included in the article's Creative Commons licence, unless indicated otherwise in a credit line to the material. If material is not included in the article's Creative Commons licence and your intended use is not permitted by statutory regulation or exceeds the permitted use, you will need to obtain permission directly from the copyright holder. To view a copy of this licence, visit http://creativecommons.org/licenses/by/4.0/ The Creative Commons Public Domain Dedication waiver (http://creativecommons.org/publicdomain/zero/1.0/) applies to the data made available in this article, unless otherwise stated in a credit line to the data. 
multiple forms of CAM have been shown to interfere with chemotherapy regimens $[8,9]$.

Growing awareness of the prevalence of traditional medicine use has spurred biomedical efforts to test its effectiveness. Promisingly, a number of Nigerian herbal medicines have been tested and have demonstrated efficacy against malaria [10]. However, traditional medicine usage has also been shown to interfere with proper adherence to biomedical treatment regimes. Previous studies have found relationships between traditional medicine usage and medication non-adherence [4], and many patients turn to biomedical treatment only after traditional medicine has failed to cure them [11]. Unfortunately, a majority of patients do not disclose their use of traditional medicine to biomedical doctors, in large part because doctors fail to ask $[2,12]$. A recent study of American oncologists found that only $26 \%$ initiate discussions about herbs and supplements with their patients, and that they significantly underestimate the percentage of their patients who use CAM. In reality, there is high CAM utilization among cancer patients ranging from 43 to $88 \%$, and these numbers may be even higher in lower-income countries [13-15].

Despite the popularity of traditional medicine in Nigeria, there are few regulations on its use or quality control. One study of herbal medicines in Nigeria found that $100 \%$ of herbal medicine samples contained elevated amounts of heavy metals such as cadmium, lead, and mercury [16]. Other research has revealed levels of microbial contamination of herbal medicines that are unacceptably high by WHO standards [17]. This is concerning, as herbal medicine usage is widespread, and widely regarded as safe by its users [1].

Usage of traditional medicine is often attributed to its relative ease of access and affordability when compared to biomedicine. This is particularly relevant in the African continent, where the ratio of traditional healers to population can be 100 times greater than that of medical doctors to population [18]. A previous study in Ibadan, Nigeria found that people were more likely to choose traditional medicine because of perceived higher effectiveness, accessibility, and affordability [19]. Another cross-sectional study identified increased utilization of $\mathrm{TM}$, and corresponding decreased utilization of biomedicine, in populations who believe in supernatural causes of disease [20].

While many studies have been performed on the use of traditional medicines in patients with a variety of health conditions, few have investigated the use of traditional medicine by healthy Nigerian adults [1]. Previous studies have identified influence of family or friends as an important determinant of traditional medicine use [1, 6]. However, there have been conflicting findings as to the importance of age, socioeconomic status, and education level in predicting an individual's usage of traditional medicine [4, 5, 21]. Given both the prevalence of traditional medicine use among Nigerian populations, and potential danger of consuming contaminated herbal medicines, it is important to understand patterns of usage. This study aims to identify the prevalence of, and determinants of TM use among healthy adult women in the urban city of Ibadan, Nigeria.

\section{Methods \\ Subjects}

Seven hundred fourty-eight adult women were recruited using community-based methods from Ibadan, Nigeria from 2013 to 2015 as healthy controls for the Nigerian Breast Cancer Study (NBCS), a case-control study of breast cancer. These controls were recruited to match breast cancer cases recruited in the surgery and oncology departments according to Nigerian ethnicity and age group. The subjects were all adult females who were 18 years or older, absent of breast cancer, and able to give informed consent. The Yoruba participants were mainly recruited from the Akinyele LGA of Oyo State (Oboda, Sawmill, Abiola Quarters, Tybato, Aregbe, Idi-aba and Idi-Ose), where recruitment was done by house visits. The Hausa participants were recruited from communities in Sabo, and women interested in participating came to the Sabo Palace and were interviewed with the help of an interpreter. The Ibo and other ethnic group participants were recruited from Aleshiloye market, a major market in Ibadan. The recruitment methods and study design have been documented in detail elsewhere [22, 23]. All subjects were informed about the research and gave consent. This project was approved by institutional review boards at the University of Chicago and the University of Ibadan.

\section{Questionnaire}

Interviews were conducted in person as part of epidemiological and demographic data collection for NBCS. Participants were informed that the questionnaire would ask about a variety of demographic and health-related factors. Interviews were conducted in either English or Yoruba by trained researchers. The questionnaire collected demographic data such as age, education level, ethnicity, and socioeconomic status, as well as healthrelated data such as body mass index (BMI), which was categorized into underweight $(<18.5)$, normal $(18.5-$ 24.9), overweight (25.0-29.9), and obese (30+) according to the World Health Organization categorizations [24]. Women were asked how their weight compared to their weight 1 year prior. Women were also asked whether or not they had used traditional medicine, the frequency of their usage, and their reasons for using it. Respondents could choose from several multiple-choice options to 
provide their reasons, and were also allowed to specify other reasons.

\section{Statistical analyses}

Data from the questionnaire were analyzed using STATA 14.1 (StataCorp, College Station, TX, USA). Respondents were categorized into traditional medicine users and non-users. Data on income, age, BMI, and education were re-classified into appropriate categories. Subjects' reported income was converted from Nigerian Naira into US Dollars based on the currency exchange rates at the month and year of each subject's interview. Descriptive statistics were generated and bivariate analyses using Chi-Square and Wilcoxon rank-sum tests were conducted to determine the factors that were significantly associated with TM use. Logistic regression models were then run to identify determinants of TM use among women in the study. Post-estimation tests and trend tests were then conducted to provide information about the global significance of each variable after running the multiple logistic regression. This global test is appropriate before pair-wise comparison. The normal or majority group was used as the referent group in the calculation of odds ratio (OR) and 95\% confidence interval (CI).

\section{Results}

\section{Subject characteristics}

Seven hundred fourty-eight adult women were interviewed, with an average age of 42.7 years (range: $18-90$ years; SD 14.3). Women reported a median total household monthly income of $\$ 94.7$ adjusted USD (range: \$0-\$1594) and a majority had an education level of primary school or below. Most subjects belonged to the Yoruba ethnic group (66.7\%), with Hausa (22.3\%) and Ibo (4.0\%) comprising the largest minority groups. A majority of women reported their occupation as trader (63.8\%), with artisan being the second most common occupation (12.2\%).

\section{Usage of traditional medicine}

Six hundred women (81.6\%) reported having taken TM and 135 (18.4\%) reported not having ever taken TM. The reasons for taking TM were varied, with the most commonly reported reasons being fever (40\%) and Jedijedi/Pile (29\%), known as hemorrhoids in the biomedical literature. Table 1 shows the most commonly reported reasons for taking TM. Of those who took TM, only a minority reported taking it everyday (14.3\%). Rather, the most frequently reported frequency of use was less than once a week $(36.5 \%)$, with $31.1 \%$ of those women taking TM less than once a week, but more than once a month-the median frequency of usage. Table 2 summarizes the reported frequency of usage for TM users.
Table 1 Top Reasons for Taking Traditional Medicine ${ }^{a}$

\begin{tabular}{|c|c|c|}
\hline Reason & Number & Percentage $(95 \% \mathrm{Cl})$ \\
\hline Fever & 340 & $40.0(36.8-43.5)$ \\
\hline Jedi-jedi/Pile & 245 & $28.9(25.8-32.0)$ \\
\hline Malaria & 46 & $5.41(3.99-7.15)$ \\
\hline Pregnancy & 32 & $3.76(2.59-5.27)$ \\
\hline Stomachache & 30 & $3.53(2.39-5.00)$ \\
\hline Prevention of Disease & 20 & $2.35(1.44-3.61)$ \\
\hline Backache & 15 & $1.76(0.99-2.89)$ \\
\hline Typhoid Fever & 15 & $1.76(0.99-2.89)$ \\
\hline Hypertension & 11 & $1.29(0.65-2.30)$ \\
\hline Dysentery & 9 & $1.05(0.49-2.00)$ \\
\hline Headache & 8 & $0.94(0.41-1.84)$ \\
\hline Diabetes & 7 & $0.82(0.33-1.69)$ \\
\hline Fertility & 7 & $0.82(0.33-1.69)$ \\
\hline Body Ache & 6 & $0.71(0.26-1.53)$ \\
\hline Ulcer & 5 & $0.59(0.19-1.37)$ \\
\hline Body Weakness & 4 & $0.47(0.13-1.20)$ \\
\hline Leg Pain & 4 & $0.47(0.13-1.20)$ \\
\hline Other Diseases & 4 & $0.47(0.13-1.20)$ \\
\hline Menstrual Problems & 4 & $0.47(0.13-1.20)$ \\
\hline Anemia & 3 & $0.35(0.07-1.03)$ \\
\hline Asthma & 3 & $0.35(0.07-1.03)$ \\
\hline Chest Pain & 3 & $0.35(0.07-1.03)$ \\
\hline Diarrhea & 3 & $0.35(0.07-1.03)$ \\
\hline Toothache & 3 & $0.35(0.07-1.03)$ \\
\hline Cold & 2 & $0.24(0.03-0.85)$ \\
\hline Fibroid & 2 & $0.24(0.03-0.85)$ \\
\hline Neck Pain & 2 & $0.24(0.03-0.85)$ \\
\hline Laxative & 2 & $0.24(0.03-0.85)$ \\
\hline Rash & 2 & $0.24(0.03-0.85)$ \\
\hline Cough & 2 & $0.24(0.03-0.85)$ \\
\hline Arthritis & 1 & $0.12(0.00-0.65)$ \\
\hline Breast Pain & 1 & $0.12(0.00-0.65)$ \\
\hline Deworming & 1 & $0.12(0.00-0.65)$ \\
\hline Epilepsy & 1 & $0.12(0.00-0.65)$ \\
\hline Mastitis & 1 & $0.12(0.00-0.65)$ \\
\hline Finger Numbness & 1 & $0.12(0.00-0.65)$ \\
\hline Tumor & 1 & $0.12(0.00-0.65)$ \\
\hline Dizziness & 1 & $0.12(0.00-0.65)$ \\
\hline Throat Pain & 1 & $0.12(0.00-0.65)$ \\
\hline Oily Food Protection & 1 & $0.12(0.00-0.65)$ \\
\hline Well-being & 1 & $0.12(0.00-0.65)$ \\
\hline
\end{tabular}

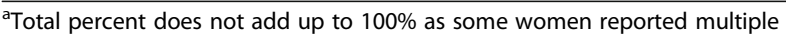
reasons for TM use 
Table 2 Frequency of Taking Traditional Medicines

\begin{tabular}{lll}
\hline Frequency of Usage & Freq. & Percent \\
\hline Everyday & 86 & 14.3 \\
2-6 times/week & 68 & 11.3 \\
Once a week & 46 & 7.7 \\
< Once a week, & 99 & 16.5 \\
but $\geq$ once a month & & \\
<Once a month & 219 & 36.5 \\
Don't Know & 82 & 13.7 \\
Total & 600 & 100 \\
\hline
\end{tabular}

\section{Relationship between demographic factors and} traditional medicine usage

Table 3 displays demographic characteristics of the women in the study and results of bivariate analyses. Ethnicity, education, occupation, and weight change were statistically significant in the analysis. Women who had been educated were generally less likely to use TM than those who had not had an education, and women who had a secondary education were significantly less likely to use TM than their non-educated counterparts. In addition, women who were traders, the most common occupation, were generally more likely than other occupations to use TM, and significantly so when compared with artisans. The relationship between income and TM usage bordered on significance, and women with the highest total household monthly incomes were more likely to use TM than those with the lowest incomes. No relationship was found between TM usage and age, marital status, or BMI in the bivariate analysis. This lack of significance persisted even after controlling for other demographic factors in a logistic regression.

A logistic model, shown in Table 4, was created using significant predictors identified from the bivariate analyses. Income was excluded from the analysis due to maximize sample size and due to the strong correlation between income and ethnicity. Ethnicity, education and weight change remained significant predictors for TM use. Most strikingly, women of Ibo or Hausa were significantly less likely to use TM than women of Yoruba. There was also a positive relationship between TM use and weight loss, with women who reported significant weight loss over the past year significantly more likely to use TM than those whose weight had not changed, and even more so than those who had gained weight. In addition, more educated women were less likely to use TM. Occupation lost statistical significance after controlling for other factors in the logistic regression.

\section{Discussion}

This was the first known study examining the usage of traditional medicine among adult women in Nigeria. Most other studies have focused on TM usage among
Table 3 Demographics of Traditional Medicine Users and NonUsers

\begin{tabular}{|c|c|c|c|c|}
\hline Demographics & TM Users n (\%) & Non-Users n (\%) & $n$ & $P$-value \\
\hline \multicolumn{5}{|l|}{ Monthly Income } \\
\hline$<\$ 40$ & $129(75.9 \%)$ & 41 (24.1\%) & \multirow[t]{4}{*}{671} & \multirow[t]{4}{*}{0.051} \\
\hline$\$ 40-\$ 100$ & 160 (84.7\%) & $29(15.3 \%)$ & & \\
\hline$\$ 100-\$ 160$ & $130(82.8 \%)$ & $27(17.2 \%)$ & & \\
\hline$\$ 160+$ & $132(85.2 \%)$ & $23(14.8 \%)$ & & \\
\hline \multicolumn{5}{|l|}{ Age Category } \\
\hline $17-29$ & $90(73.2 \%)$ & $33(26.8 \%)$ & \multirow[t]{5}{*}{731} & \multirow[t]{5}{*}{0.13} \\
\hline $30-39$ & $194(84.4 \%)$ & 36 (15.7\%) & & \\
\hline $40-49$ & 124 (81.6\%) & $28(18.4 \%)$ & & \\
\hline $50-59$ & $81(82.7 \%)$ & $17(17.3 \%)$ & & \\
\hline $60+$ & 107 (83.6\%) & $21(16.4 \%)$ & & \\
\hline \multicolumn{5}{|l|}{ Education } \\
\hline None & $116(88.5 \%)$ & $15(11.5 \%)$ & \multirow[t]{5}{*}{688} & \multirow[t]{5}{*}{0.014} \\
\hline Primary & $272(83.7 \%)$ & $53(16.3 \%)$ & & \\
\hline Secondary & $114(75.0 \%)$ & $38(25.0 \%)$ & & \\
\hline Vocational/Tech & 15 (71.4\%) & $6(28.6 \%)$ & & \\
\hline College and Above & $46(78.0 \%)$ & $13(22.0 \%)$ & & \\
\hline \multicolumn{5}{|l|}{ Ethnicity } \\
\hline Yoruba & $423(86.7 \%)$ & 65 (13.3\%) & \multirow[t]{4}{*}{733} & \multirow[t]{4}{*}{$<0.001$} \\
\hline Ibo & $16(55.2 \%)$ & $13(44.8 \%)$ & & \\
\hline Hausa & $122(73.5 \%)$ & $44(26.5 \%)$ & & \\
\hline Other & $37(74.0 \%)$ & $13(26.0 \%)$ & & \\
\hline \multicolumn{5}{|l|}{ Occupation } \\
\hline None & $34(77.3 \%)$ & $10(22.7 \%)$ & \multirow[t]{7}{*}{719} & \multirow[t]{7}{*}{0.011} \\
\hline Housewife & $44(78.6 \%)$ & $12(21.4 \%)$ & & \\
\hline Trader & 391 (85.4\%) & $67(14.6 \%)$ & & \\
\hline Farmer & $4(100 \%)$ & $0(0 \%)$ & & \\
\hline Artisan & $67(75.3 \%)$ & $22(24.7 \%)$ & & \\
\hline Professional & $35(81.4 \%)$ & $8(18.6 \%)$ & & \\
\hline Other & $15(60.0 \%)$ & $10(40.0 \%)$ & & \\
\hline \multicolumn{5}{|l|}{ Current BMI } \\
\hline Underweight & $28(84.8 \%)$ & $5(15.2 \%)$ & \multirow[t]{4}{*}{730} & \multirow[t]{4}{*}{0.93} \\
\hline Normal & 221 (81.5\%) & $50(18.5 \%)$ & & \\
\hline Overweight & $180(80.4 \%)$ & 44 (19.6\%) & & \\
\hline Obese & $166(82.2 \%)$ & $36(17.8 \%)$ & & \\
\hline \multicolumn{5}{|l|}{ Marital Status } \\
\hline Married & $487(83.4 \%)$ & 97 (16.6\%) & \multirow[t]{4}{*}{731} & \multirow[t]{4}{*}{0.082} \\
\hline Widowed & $81(75.0 \%)$ & $27(25.0 \%)$ & & \\
\hline Divorced/Separated & $10(83.3 \%)$ & $2(16.7 \%)$ & & \\
\hline Never Married & $19(70.4 \%)$ & $8(29.6 \%)$ & & \\
\hline \multicolumn{5}{|l|}{ Weight Change } \\
\hline Significant Gain & $15(53.6 \%)$ & $13(46.4 \%)$ & \multirow[t]{5}{*}{732} & $<0.001$ \\
\hline Little Gain & $100(74.1 \%)$ & $35(25.9 \%)$ & & \\
\hline About the same & $320(84.4 \%)$ & $59(15.6 \%)$ & & \\
\hline Little Loss & $144(85.2 \%)$ & $25(14.8 \%)$ & & \\
\hline Significant Loss & 18 (85.7\%) & $3(14.3 \%)$ & & \\
\hline
\end{tabular}

Reported $p$-values were calculated from Wilcoxon Rank-Sum tests for Income, Age, Education, BMI, and Weight Change (over the last year) and from Chi-Square tests for Occupation, Marital Status and Ethnicity. 
Table 4 Multivariable Logistic Regression of Demographic Factors and Traditional Medicine Use $(N=668, p<0.001)$

\begin{tabular}{|c|c|c|}
\hline Variables & Odds Ratio (95\% Cl) & $p$-value \\
\hline \multicolumn{3}{|c|}{ Education } \\
\hline None & 1 (ref) & 0.039 \\
\hline Primary & $0.75(0.38-1.46)$ & \\
\hline Secondary & $0.42(0.21-0.85)$ & \\
\hline Vocational/Tech & $0.40(0.12-1.32)$ & \\
\hline College and Above & $0.53(0.19-1.49)$ & \\
\hline \multicolumn{3}{|c|}{ Ethnicity } \\
\hline Yoruba (ref) & 1 (ref) & $<0.001$ \\
\hline Ibo & $0.25(0.10-0.63)$ & \\
\hline Hausa & $0.43(0.24-0.76)$ & \\
\hline Other & $0.50(0.24-1.02)$ & \\
\hline \multicolumn{3}{|c|}{ Occupation } \\
\hline Trader (ref) & 1 (ref) & 0.076 \\
\hline None & $0.56(0.24-1.27)$ & \\
\hline Housewife & $0.91(0.40-2.08)$ & \\
\hline Farmer & (dropped) & \\
\hline Artisan & $0.55(0.31-0.97)$ & \\
\hline Professional & $1.05(0.37-2.96)$ & \\
\hline Other & $0.33(0.13-0.85)$ & \\
\hline \multicolumn{3}{|c|}{ Weight Change } \\
\hline Significant gain & $0.34(0.13-0.87)$ & 0.006 \\
\hline Little gain & $0.69(0.40-1.17)$ & \\
\hline No change (ref) & 1.00 (ref) & \\
\hline Little loss & $1.20(0.68-2.10)$ & \\
\hline Significant loss & $1.64(0.44-6.09)$ & \\
\hline
\end{tabular}

$P$-values for testing global association of each variable were generated from post-estimation tests, with the weight change (over the last year) and education variables tested using a trend test

those with specific illnesses. The rate of usage found in this study, $81.6 \%$, is consistent with that found in other studies of general populations in Nigeria (66.8-84.7\%) $[1,25,26]$. The most common reasons for using TM in this study were fever (40\%) and Jedi-jedi/pile (29\%). Other studies have found malaria to be a common reason, and an herbal preparation known as 'Agbo jedi-jedi' to be the most common medicine used [1].

Although this study did not specifically examine TM use in malaria, prior studies have found self-diagnosis and self-medication to be the most common form of diagnosis and treatment for malaria [27-29]. Fevers are often presumed by laypeople to be diagnostic for malaria, which may be problematic because self-diagnoses are more likely to be inaccurate [29]. As two of the top three reasons for using TM were fever and malaria, it is possible that the women in this study were engaging in self-medication without seeking professional medical consultations.

The most striking finding was the inter-ethnic group differences in rates of TM usage. After controlling for other factors, those identifying with the Ibo ethnic group were four times less likely to use traditional medicine than the Yoruba ethnic group $(\mathrm{OR}=0.25)$. Those belonging to the Hausa ethnic group were about half as likely to use traditional medicine than the Yoruba group $(\mathrm{OR}=0.43)$. To our knowledge, this is the first study showing that usage of traditional medicines varied across ethnicities.

As Ibadan, the site of this study, is a majority Yoruba city, individuals from other ethnic groups are most likely immigrants from other areas. This precludes us from generalizing our conclusions to the general Hausa or Ibo populations, as there may be differences between minorities who immigrated to Ibadan and those who stayed in their hometown. In addition, when income is included in the regression model, the significance of ethnicity decreases, suggesting that income differences among the ethnicities may account for some of the differences in TM usage. However, it is important to note that the effect of ethnicity remained significant $(p=0.02)$ even after controlling for factors such as education, income, and occupation. A recent study found that belief in supernatural causes of disease is associated with increased TM usage [20]. It may be possible that beliefs towards health and illness differ among the ethnic groups, thus affecting attitudes and usage of traditional medicine. Now that ethnicity has been identified as a significant determinant, further studies are needed to investigate the reasons behind these differences.

Although past studies have not found a relationship between education level and traditional medicine use [2], our study showed a significant trend towards highly educated women being less likely to use traditional medicine than those with no education, with a significant effect found for women with a secondary education $(\mathrm{OR}=0.42)$. This may be a function of a previously described relationship between education and views on harmful side effects of herbal medicine [21]. Because highly educated women may be more likely to believe that herbal medicine has harmful side effects, they may be less likely to use it. Although previous studies have found a relationship between marital status and traditional medicine use [4], we did not find a significant relationship in our study.

Given that affordability is often cited as a major reason that people use TM rather than biomedicine, we expected to see a relationship between income and TM usage [19]. However, the trend in this study was the 
opposite, and women with higher total household monthly incomes were more likely to use traditional medicine than those with lower incomes. Although this was not significant, it suggests that affordability may not be the major factor in women's decision to use TM. It is noteworthy to contrast the inverse relationship between education levels and TM use and the positive relationship between income levels and TM use. Women who are richer are more likely to use TM, as are women who are less educated.

Previous research has found a positive correlation between increasing BMI and herbal medicine use [5]. However, these results were not corroborated in our study, in which current BMI had no significant association with TM use. Interestingly, what did seem to matter was women's weight change over the past year. Women who gained significant weight over the past year were three times less likely to use traditional medicine $(\mathrm{OR}=0.32)$ when compared to women whose weights did not change. In contrast, the likelihood of using traditional medicine was highest among women who had lost significant weight in the past year (85.7\%), and there was a significant association between increasing amounts of weight loss and increased usage of traditional medicine. Women may have experienced weight gain from an improvement in health, resulting in a lower propensity to use traditional medicine. On the other hand, significant weight loss may have been indicative of a major illness, or usage of traditional medicines targeted towards weight loss.

This study had multiple limitations. The questionnaire did not differentiate between herbal medicines and other spiritual or healing practices that may be considered traditional medicine. A further limitation is that we could not determine whether women were taking medicine at the suggestion of an herbal medicine specialist, or if they obtained medicines after self-diagnosis. In addition, there were no questions about the types of medicines used, nor about attitudes and beliefs towards traditional medicine. This limits our ability to draw conclusions about why ethnicity, education, and weight change seem to affect women's propensity to take traditional medicine. Finally, the community-based recruitment method may have resulted in a sample that is not fully representative of the general population.

The finding that large numbers of women in the general population use traditional medicine is significant for multiple reasons. Chemical analyses of commonly used herbal products have found concerning levels of toxicity and contamination. Given the high rates of traditional medicine use in the general population, it is imperative that more research is done to investigate the safety of these products. In addition, previous studies have found significant associations between traditional medicine use and medication noncompliance [30], but that most patients did not mention traditional medicine use to their doctors [2]. As it is likely that their patients are using traditional medicine, doctors in Nigeria should ask their patients about TM usage as part of the standard interview. Our study only included the healthy controls from the NBCS. However, future studies should examine rates of TM usage among breast cancer patients, and determine whether or not TM usage affects adherence to drug and chemotherapy regimens, as seen in other studies [30].

\section{Conclusions}

In conclusion, our study found a high rate of TM usage among women living in a major urban center in Nigeria consistent with that found in prior studies. This is significant because of the prevalence of contaminated herbal products, the association between TM usage and noncompliance with biomedical treatment regimens. The most striking finding was that ethnicity was significantly correlated with traditional medicine use, with the Ibo and Hausa ethnic groups less likely to use traditional medicine than their Yoruba counterparts. Other departures from previous research include the finding that education was a significant determinant of TM use, and that income was not significant. Future studies should explore the reasons behind differential usage in different ethnic groups, investigate how attitudes and beliefs affect usage rates, and further investigate how TM usage affects medical treatment. TM usage is widespread among the general population in Nigeria, and is likely just as, if not more common among patients with cancer or other illnesses. Given the risks of interference with medication regimens and the prevalence of contaminated herbal products, physicians should regularly engage in conversations with their patients about their TM usage.

\section{Supplementary information}

Supplementary information accompanies this paper at https://doi.org/10. 1186/s12906-020-02881-z.

Additional file 1. The original questionnaire used to collect data for this study is included here.

Additional file 2. The cover letter for the BMC Complementary Alternative Medicine journal is included here.

\section{Abbreviations}

TM: Traditional medicine; OR: Odds ratio; WHO: World Health Organization; CAM: Complementary and alternative medicine; NBCS: Nigerian breast cancer study; UCH: University College Hospital; BMI: Body mass index; $\mathrm{Cl}$ : Confidence interval

\section{Acknowledgments}

The authors are indebted to the participants of the NBCS, UCH hospital staff, and research assistants for their cooperation and dedication to this project. Special gratitude goes to Frank Qian for his statistical expertise and Chibuzor Nkwodimmah for her assistance in data collection and interpretation. 


\section{Author's contributions}

SL conceived of the project idea, analyzed and interpreted the data and wrote the manuscript. SO and IA collected the data and contributed to the interpretation of results. $\mathrm{OO}$ helped supervise the project. $\mathrm{DH}$ and $\mathrm{OIO}$ were involved in the conception of the project idea, analysis and interpretation of data, and supervised the project. All authors discussed the results and contributed to the final manuscript. The authors read and approved the final manuscript.

\section{Funding}

This work was supported by American Cancer Society (MRSG-13-063-01-TBG, CRP-10-119-01-CCE), National Cancer Institute (CA161032), Susan G. Komen for the Cure, Breast Cancer Research Foundation and the Pritzker Summer Research Program. The funders had no role in study design, data collection and analysis, decision to publish, or preparation of the manuscript.

\section{Availability of data and materials}

The datasets generated and analyzed during the current study are not publicly available due to the presence of protected health information but are available from the corresponding author on reasonable request.

\section{Ethics approval and consent to participate}

Written informed consent was obtained from all participants prior to survey administration. This project was approved by institutional review boards at the University of Chicago (13304B) and the University of Ibadan (UI/IRC/02/ 003).

\section{Consent for publication}

Not applicable.

\section{Competing interests}

The authors declare that they have no competing interests. No financial interest or benefit will arise from the direct applications of this research.

\section{Author details}

'Department of Internal Medicine, Massachusetts General Hospital, Boston, MA, USA. ${ }^{2}$ Center for Population and Reproductive Health, College of Medicine, University of Ibadan, UCH, Ibadan, Nigeria. ${ }^{3}$ Department of Public Health Sciences, University of Chicago, Chicago, IL, USA. ${ }^{4}$ Center for Clinical Cancer Genetics and Global Health, Department of Medicine, University of Chicago, Chicago, IL, USA.

Received: 15 June 2019 Accepted: 5 March 2020

Published online: 20 March 2020

\section{References}

1. Oreagba I, et al. Herbal medicine use among urban residents in Lagos, Nigeria. BMC Complement Altern Med. 2011;11:117.

2. Ezeome E, Anarado A. Use of complementary and alternative medicine by cancer patients at the University of Nigeria Teaching Hospital, Enugu, Nigeria. BMC Complement Altern Med. 2007;7:28.

3. Ogbera $\mathrm{AO}$, et al. Complementary and alternative medicine use in diabetes mellitus. West Afr J Med. 2010;29(3):158-62.

4. Namuddu B, et al. Prevalence and factors associated with traditional herbal medicine use among patients on highly active antiretroviral therapy in Uganda. BMC Public Health. 2011;11:855.

5. Amira O, Okubadejo N. Frequency of complementary and alternative medicine utilization in hypertensive patients attending an urban tertiary care centre in Nigeria. BMC Complement Altern Med. 2007:7:30

6. Oshikoya $\mathrm{K}$, et al. Use of complementary and alternative medicines for children with chronic health conditions in Lagos, Nigeria. BMC Complement Altern Med. 2008;8:66.

7. Adepoju J. A study of health beliefs and practices of the Yoruba. J Cult Divers. 2012;19(2):36-43.

8. Ben-Arye $\mathrm{E}$, et al. Potential risks associated with traditional herbal medicine use in cancer care: a study of middle eastern oncology health care professionals. Cancer. 2016;122(4):598-610

9. Oneschuk D, Younus J. Natural health products and cancer chemotherapy and radiation therapy. Oncol Rev. 2008;1:233-42.

10. Lawal B, et al. Potential antimalarials from African natural products: a review. J Intercult Ethnopharmacol. 2015;4(4):318-43.
11. Asuzu C, et al. A pilot study of cancer patients' use of traditional healers in the radiotherapy department, university college hospital, Ibadan, Nigeria. Psychooncology. 2015;26(3):369-76.

12. Davis $\mathrm{E}_{\text {, et }}$ al. Cancer patient disclosure and patient-doctor communication of complementary and alternative medicine use: a systematic review. Oncologist. 2012;17(11):1475-81.

13. Ge J, et al. Patient-physician communication about complementary and alternative medicine in a radiation oncology setting. Int J Radiat Oncol Biol Phys. 2013;85(1):e1-6.

14. Dioro $C$, et al. Global use of traditional and complementary medicine in childhood cancer: a systematic review. J Glob Oncol. 2017:3(6):791-800.

15. Lee R, et al. National survey of US oncologists' knowledge, attitudes, and practice patterns regarding herb and supplement use by patients with cancer. J Clin Oncol. 2014;32(36):4095-101.

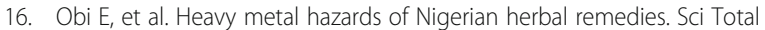
Environ. 2006;369:35-41.

17. Ezekwesili-Ofili J. Et. al bioload and aflatoxin content of herbal medicines from selected states in Nigeria. Afr J Tradit Complement Altern Med. 2014 11(3):143-7.

18. Krah E, de Kruijf J, Ragno L. Integrating traditional healers into the health care system: challenges and opportunities in rural northern Ghana. J Community Health. 2018:43(1):157-63.

19. Adejumo A, Faluyi M, Adejuwon A. Role of socio-psychological factors in perceived quality of care rendered by traditional medical practitioners in Ibadan, Nigeria. Glob J Health Sci. 2013;5(6):186-96.

20. Chukwuneke F, et al. Culture and biomedical care in Africa: the influence of culture on biomedical care in a traditional African society, Nigeria, West Africa. Niger J Med. 2016;21(3):331-3.

21. Fakeye T, Adisa R, Musa I. Attitude and use of herbal medicines among pregnant women in Nigeria. BMC Complement Altern Med. 2009;9:53.

22. Sighoko D, Ogundiran T, Ademola A, et al. Breast cancer risk after full-term pregnancies among African women from Nigeria, Cameroon, and Uganda. Cancer. 2015:121(13):2237-43.

23. Hou N, Ndom P, Jombwe J, et al. An epidemiologic investigation of physical activity and breast cancer risk in Africa. Cancer Epidemiol Biomark Prev. 2014;23(12):2748-56

24. World Health Organization. Body Mass Index - BMI. Retrieved from http:// www.euro.who.int/en/health-topics/disease-prevention/nutrition/a-healthylifestyle/body-mass-index-bmi. Accessed 23 Oct 2018.

25. Okoronkwo l, et al. Patterns of complementary and alternative medicine use, perceived benefits, and adverse effects among adult users in Enugu urban, Southeast Nigeria. Evid Based Complement Altern Med. 2014;2014: 239372.

26. Onyiapat J, Okoronkwo I, Ogbonnaya N. Complementary and alternative medicine use among adults in Enugu, Nigeria. BMC Complement Altern Med. 2011;11:19.

27. Ansumana R, et al. Presumptive self-diagnosis of malaria and other febrile illnesses in Sierra Leone. Pan Afr Med J. 2013;15:34.

28. Uzochukwu B, Onwujekwe O. Socio-economic differences and health seeking behaviour for the diagnosis and treatment of malaria: a case study of four local government areas operating the Bamako initiative programme in south-east Nigeria. Int J Equity Health. 2003;3:6.

29. Nsagha D, et al. Knowledge and practices relating to malaria in a semiurban area of Cameroon: choices and sources of antimalarials, selftreatment and resistance. Pan Afr Med J. 2011;9:8.

30. Boima $V$, et al. Factors associated with medication nonadherence among hypertensives in Ghana and Nigeria. Int J Hypertens. 2015;2015:205716.

\section{Publisher's Note}

Springer Nature remains neutral with regard to jurisdictional claims in published maps and institutional affiliations. 